\title{
A Survey on Handwritten Character Recognition using Deep Learning Technique
}

\author{
Thatikonda Somashekar \\ Research Scholar, Department of Computer Science \& Engineering, University College of Engineering(A), \\ Osmania University Hyderabad, Telangana, India \\ Email: soma.ts@gmail.com
}

Abstract-Due to its broad range of applications, handwritten character recognition is widespread. Processing application forms, digitizing ancient articles, processing postal addresses, processing bank checks, and many other handwritten character processing fields are increasing in popularity. Since the last three decades, handwritten characters have drawn the attention of researchers. For successful recognition, several methods have been suggested. This paper presents a comprehensive overview of handwritten character recognition using a neural network as a machine learning tool.

Keywords-ANN, Feature Extraction, Classification, Handwritten Character Recognition, Machine learning, $S V M$

\section{INTRODUCTION}

Handwritten Character Recognition (HCR) is a classic pattern recognition application, to begin with; Bezdek et al. described pattern recognition as recognizing structure in data by comparisons to known systems. The available system is created through a classification method. Handwritten character recognition, in general, is the method of classifying characters from handwritten input texts into predefined character groups. HCR has a wide range of applications, including character recognition, character recognition, character recognition, character recognition, character recognition, character recognition, character recognition, character recognition. Handwritten records are digitized. Reading the application form and making decisions based on the Unknown language's data is recognized and translated into a known language by a translation device. Blind people's reading aids, bank check handling Verification of signatures Number plates for automobiles To postal mail, for example, an automatic pin code reader is used.

Character awareness is something we do all the time in our everyday lives. Our brain constantly performs the HCR when reading notes, signs, or novels. We compare it to our previous experiences and memories and then respond, act, or infer new information. As a result, this is how we recognize characters naturally.

During, who attempted to create an aid for the visually impaired, was the first to recognize characters. In the 1940s, the first character recognizer was built. Previously, almost all works were based on machine-printed text or a limited collection of handwritten symbols or texts.

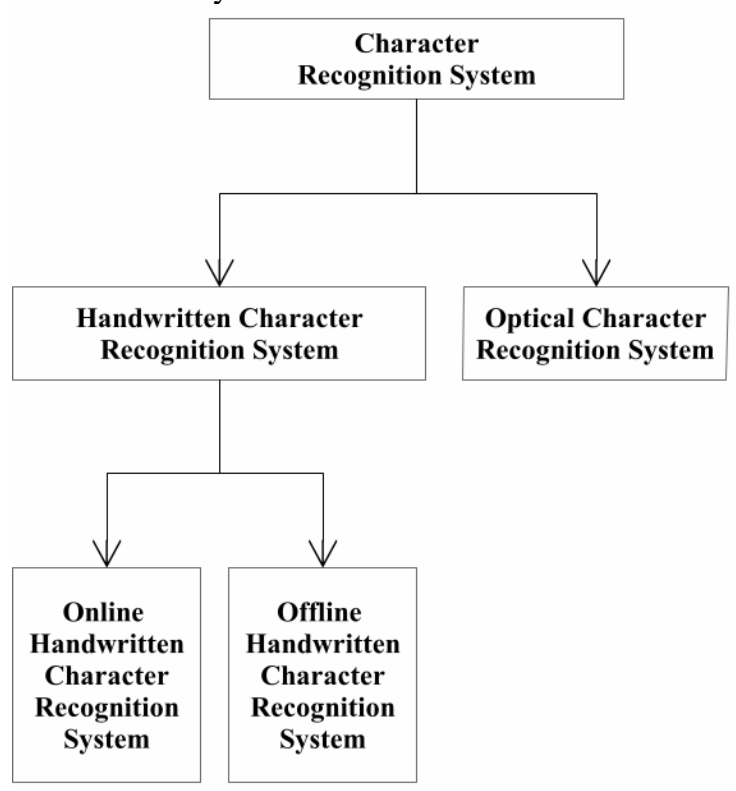

Fig. 1. Character Recognition System Types

With online and offline methods, HCR quickly gained interest in research from 1980 to 1990. After 1990, image processing and pattern recognition combined with Artificial Intelligence, resulting in the development of highly efficient and powerful computers and gadgets such as scanners, cameras, and other specialized devices. Handwritten character recognition covers a significant portion of the application space. Despite all of this testing, there is still no device that genuinely achieves the goal of handwritten character recognition. 
In general, , image acquisition, preprocessing, segmentation, feature extraction, classification phases are included in all handwritten character recognition systems (Fig 2).

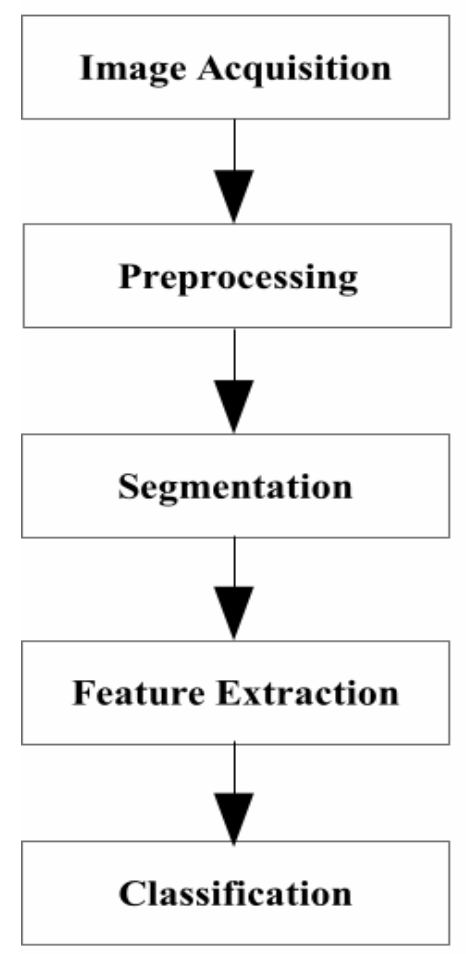

Fig. 2. The Handwritten Character Recognition System's architecture

The rest of the paper is laid out as follows. The second section summarizes the Literature on various offline handwritten character recognition systems developed by multiple researchers. The comparison table of different methods is shown in Section III. Section IV brings the survey to a close.

\section{LITERATURE SURVEY}

This study of the Literature delves into every aspect of the handwritten character recognition system.

\section{A. Image Acquisition}

Picture retrieval is the process of gathering handwritten input data for a character recognition system. Based on image or data acquisition, online and offline systems have been developed. Bluche et al. MNIST used the English-language Rimes Dataset as a numeric data standard dataset [9]. Other datasets include Chars74K (realistic images of English characters), CEDAR (paid), Semeion handwritten digit dataset, PenBased Recognition of Handwritten Digits dataset, etc. Researchers develop their recognition scheme when a standard dataset isn't available.

\section{B. Preprocessing}

The preprocessing is done on the data that has been collected. It improves the consistency of input data and makes it more appropriate for the recognition system's next step. Various techniques are used in this process, such as greyscale conversion, binary conversion, noise reduction, etc. Figure 3 depicts the image's basic preprocessing operations.

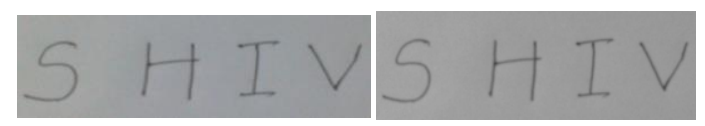

(a) RGB Image - 230x755x3 uint8 (b) Gray Scale Image - 230x755 uint8

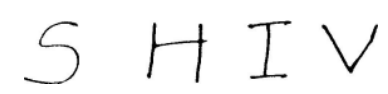

(c) Binary Image - 230x755 logical

Fig. 3. Preprocessed Images 
Bluche et al. used greyscale conversion, binary conversion, and a noise reduction technique on the input data. After considering the Grayscale and binary conversion results, the researcher used edges detection for segmentation. Otsu's algorithm is widely used for grayscale to binary image conversion.

\section{Segmentation}

Segmentation is the process of dividing an input text data image into lines and then individual characters. It gets rid of the parts of the data picture you don't like. The two types of segmentation available are external and internal segmentation. Externally, the paragraphs, lines, and words are segmented. Internal segmentation, on the other hand, is the process of separating individual characters from text input.

A variety of algorithms can be used to segment data. Histogram profiles and related component analysis are two methods for line segmentation used. Line and character after histogram-based segmentation are shown in Figures 4 and 5.

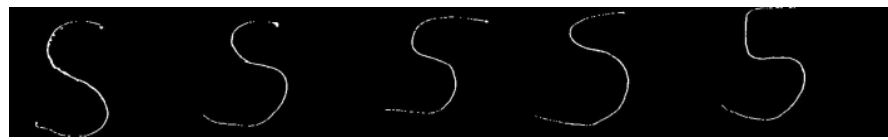

Fig. 4. Segmented Line based on Histogram

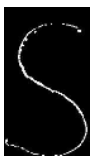

(a)

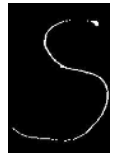

(b)

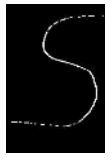

(c)

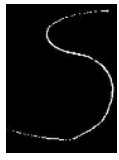

(d)

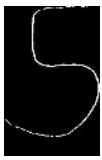

(e)

Fig. 5. Segmented Characters based on Histogram

The bounding box technique is used in character segmentation using spatial space detection for words and the histogram approach for characters and other symbols. After efficient segmentation, all segmented images are resized to ensure uniform size.

\section{Feature Extraction}

Feature extraction is the method of gathering various and beneficial information about an object or a group of things to identify new unknown objects based on that information. The robust representation of raw data is a function.

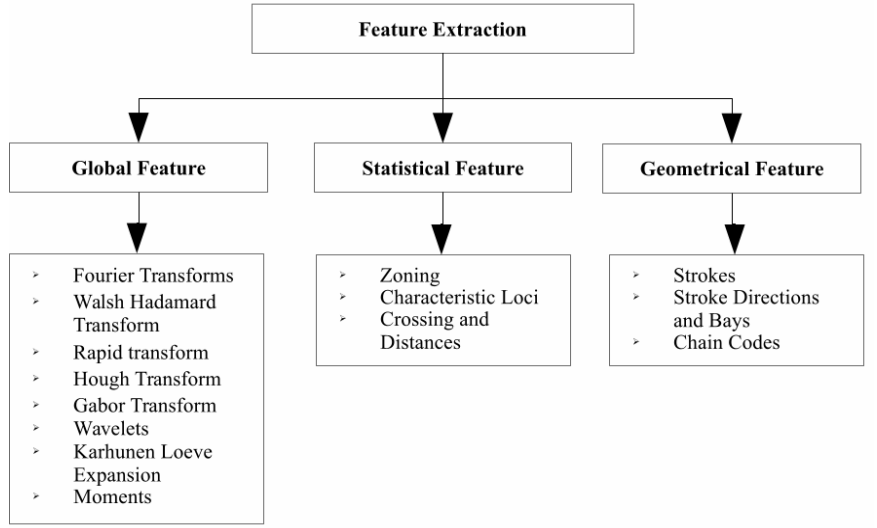

Fig. 6. Feature Extraction Techniques [22]

Various methods for extracting features are shown in Fig.6. Zone-based, statistical, structural, chain code histogram, sliding window, gradient function, hybrid, and other feature extraction techniques are among the most useful. In 1961, Freeman developed the Freeman Chain Code method of chain code. 4-neighborhood and 8-neighborhood are the most common chain code directions.

Kai Ding et al., Xuewen Wang et al., Xuewen Wang et al., Xue When comparing the Gabor feature to the Chain code (contour direction) feature, the Gabor feature outperforms the Chain code element. There are some similarities between the Gradient and Gabor features. According to Kai Ding and Cheng-Lin Liu et al., both apply to binary and grayscale images and are immune to image noise. Gabor is also better suited to large-scale texture data, even though the production is nearly identical. Imani et al. used chain code histogram function and foreground density distribution across zones. 


\section{E. Classification}

To make decisions like which class or appearance this new character belongs to, the classification or identification system is used. It means that surfaces are marked, and labels are given during the classification process. The accuracy of feature extraction and selection is critical to the classification's performance. There are various classification methods, all of which are based on image processing and artificial intelligence. Figure 7 depicts the different classification strategies. Traditional image processing techniques include template matching, statistical techniques, and structural techniques. Neural networks, fuzzy logic, and genetic techniques are examples of soft computing techniques. A hybrid delicate computing framework was proposed by Jayashree et al. She merged fuzzy logic and neural networks with an adaptive network to create neuro-fuzzy. When artificial intelligence and machine learning were combined, it covered almost all research fields, with machine learning producing outstanding results. Machine learning methods such as artificial neural networks, support vector machines, naive Bayes, nearest neighbour algorithms, decision trees, neuro-fuzzy, and others were used in a lot of handwritten character recognition.

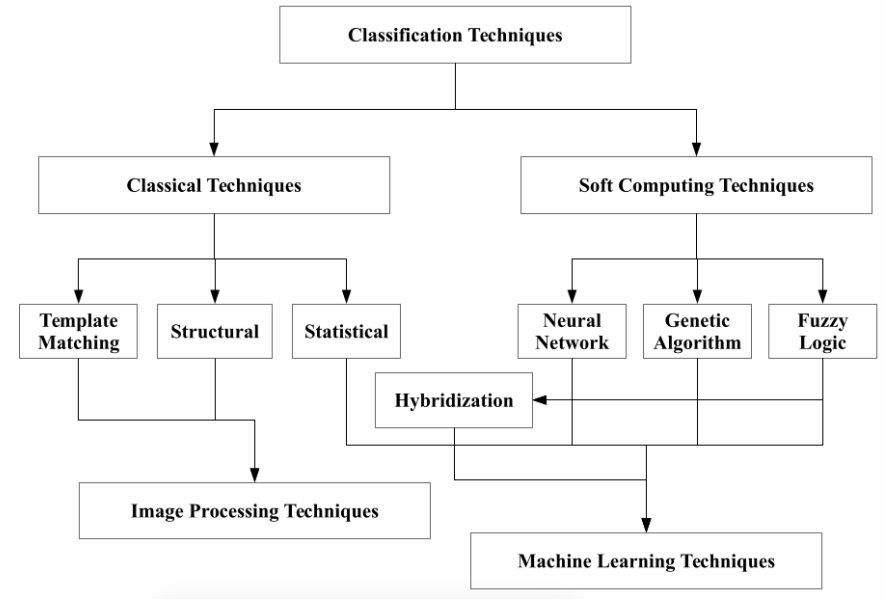

Fig. 7. Classification Techniques

Bluche et al. created an explicit feature extraction method by combining HMM with convolutional neural networks. The developed system was put to the test with the Rimes dataset, and it was discovered that while the character recognition rate was fast, the accuracy was poor. Imani and Bingyu Chi et al. used hidden Markovian Modeling, and the results show that HMM classification efficiency is more dependent on feature extraction methods.

In their proposed framework for Bangla characters, Rahman et al. used a backpropagation neural network and achieved a recognition accuracy of 94.3 percent. Backpropagation neural networks were used as a classifier by Amal Ramzi et al. in [8]. Ramzi's proposed a framework that combines online and offline functionality for handwritten Arabic characters. The proposed system had a training accuracy of 99.5 percent, but the highest recognition rate reached in testing was 78.8 percent.

The NNC (Nearest Neighbor Classifier) was compared to the SVM by Rajashekararadhya et al. (Support Vector Machine). SVM is one step ahead of NNC, according to the results. Nasien et al. combined SVM and FCC. Nasien worked with the NIST English handwritten dataset. Lowercase characters have an accuracy of 86 percent, uppercase characters have an accuracy of 88.46 percent, and (lowercase + uppercase) characters have an accuracy of 73.45 percent.

Nisha Sharma and her colleagues The proposed recognition scheme for hand-printed English characters, numerals, and special symbols used a multilayer perceptron neural network with backpropagation and an SVM classifier. Hand-printed English characters, including uppercase, lowercase, digits, and special symbols, were used as a dataset. Numbers are remembered 98 percent of the time, special characters 96.5 percent of the time, uppercase characters 95.35 percent of the time, and lowercase characters 92 percent of the time. "Neural networks are preferred because of their high noise tolerance, and SVM is preferred because of its high flexibility, scalability, and speed," Nisha Sharma et al. explained.

The following segment compares different research works in handwritten character recognition. 


\begin{tabular}{|c|c|c|c|c|c|}
\hline Paper & Feature Extraction & Language & $\begin{array}{c}\text { Data } \\
\text { set }\end{array}$ & Classifier & Results and Comments \\
\hline 1.[2] & $\begin{array}{c}\text { Simple \& Efficient } \\
\text { Zonebased } \\
\text { Hybrid } \\
\text { Feature } \\
\text { Extraction Algorithm }\end{array}$ & $\begin{array}{l}\text { Kanada } \\
\text { nd Tamil a } \\
\text { Numerals }\end{array}$ & $\begin{array}{l}\text { Numerical } \\
\text { Data Own } \\
\text { Created } \\
\text { Datasets }\end{array}$ & NNC and SVM & $\begin{array}{l}\text { Kanada }(97.75 \% \text { NNC }+98.2 \% \\
\text { SVM), } \\
\text { Tamil }(93.9 \% \text { NNC + 94.9\% SVM) }\end{array}$ \\
\hline 2.[3] & not specify & $\begin{array}{l}\text { Alphanumerical } \\
+ \text { symbols }\end{array}$ & Not specify & RNN & good recognition \\
\hline 3.[9] & $\begin{array}{l}\text { Grapheme } \\
\text { segmentation } \\
\text { Sliding Window }\end{array}$ & English & Rimes Database & MLP & Very Fast But Low accuracy \\
\hline 4.[11] & $\begin{array}{l}\text { DWTwith Multi } \\
\text { resolution technique }\end{array}$ & $\begin{array}{l}\text { English } \\
\text { Charact } \\
\text { ers }\end{array}$ & $\begin{array}{l}\text { Own } \\
\text { Character } \\
\text { Dataset }\end{array}$ & $\begin{array}{l}\mathrm{NN} \text { with } \\
\text { Euclidian } \\
\text { Distance matrix }\end{array}$ & $\begin{array}{l}\text { good accuracy - up to } 99.23 \% \text {, But } \\
\text { taking more time }\end{array}$ \\
\hline 5.[21] & $\begin{array}{l}\text { Chain Code histogram } \\
\text { Features, Distribution } \\
\text { of foreground density } \\
\text { across } \\
\text { zones }\end{array}$ & Farsi & $\begin{array}{l}\text { Own database } \\
198 \text { word } \\
\text { classes }\end{array}$ & HMM & $89.00 \%$ \\
\hline 6.[24] & Gradient feature & $\begin{array}{l}\text { Chinese } \\
\text { Alpha- } \\
\text { Numeric }\end{array}$ & $\begin{array}{l}10,000 \\
\text { gle character } \\
\text { image and } 4709 \\
\text { legal amount text } \\
\text { line images } \\
\text { extracted from } \\
\text { real } \\
\text { life Chinese bank } \\
\text { checks }\end{array}$ & HMM & Average $97.13 \%$ \\
\hline 7.[25] & $\begin{array}{l}4 \times 8 \text { and } 8 \times 4 \text { matrix for } \\
\text { each } \\
\text { chara } \\
\text { cter Segmentation of } \\
\text { column }\end{array}$ & Bangla & Not specify & NN & very simple and $94.30 \%$ \\
\hline 8.[27] & $\begin{array}{l}\text { Multi roning, } \\
\text { Geometrical feature } \\
\text { distance and angle, } \\
\text { topological feature end } \\
\text { point transition, } \\
\text { Directional feature } \\
\text { chain } \\
\text { code histogram }\end{array}$ & $\begin{array}{l}\text { English } \\
\text { characters } \\
\text { and } \\
\text { symbols }\end{array}$ & Own dataset & BPNN and SVM & $\begin{array}{l}\text { BPNN: } 98 \% \text { for English numeral, } \\
96.5 \% \text { for special characters, } \\
95.35 \% \text { for uppercase English } \\
\text { characters and } 92 \% \text { for lowercase } \\
\text { English characters SVM: } 92.167 \% \\
\text { for (uppercase and } \\
\text { lowercase)characters }\end{array}$ \\
\hline 9.[28] & $\begin{array}{l}\text { DCT Discrete } \\
\text { cosine } \\
\text { transformation }\end{array}$ & $\begin{array}{l}\text { Arabic } \\
\text { Numbers }\end{array}$ & ADBase database & $\begin{array}{l}\text { DBN - } \\
\text { Dynamic } \\
\text { Bayesian } \\
\text { Network }\end{array}$ & $\begin{array}{l}\text { Average } 85 \% \text {, this result with } \\
\text { corrupted data, slow recognition }\end{array}$ \\
\hline 10.[10] & $\begin{array}{l}7 \text { FE methods and } \\
\text { then ranking the } \\
\text { feature vector and } \\
\text { make new } 3 \text { feature } \\
\text { vector }\end{array}$ & Numeric & MNIST & $\begin{array}{l}\text { ANFIS \& } \\
\text { IBA } \\
\text { ANFIS }\end{array}$ & $\begin{array}{l}99.52 \% \text { and speed for recognition } 24 \\
\text { digits/sec }\end{array}$ \\
\hline
\end{tabular}

Table 1: Comparison Table

Comparison between various researchers proposed model shown in Table 1.

\section{DISCUSSION AND CONCLUSION}

Since it encompasses such a broad range of applications, handwritten character recognition is beneficial in our everyday lives. Several researchers proposed their work in this field, and the accuracy rate was high. Few researchers were able to concentrate and control the time complexity. After conducting extensive research, we discovered that no single technique or method could fully meet the requirements of Handwritten Character Recognition. As a result, offline handwritten character recognition remains an open research field for recognizing and resolving various complexities. 


\section{References}

[1] Timothy J Ross. Fuzzy logic with engineering applications. John Wiley \& Sons, 2009

[2] SV Rajashekararadhya and P Vanaja Ranjan. Zone-based hybrid feature extraction algorithm for handwritten numeral recognition of two popular Indian scripts. In: Nature \& Biologically Inspired Computing, 2009. NaBIC 2009. World Congress on. IEEE. 2009 pp. 526530

[3] Laurence Likforman-Sulem. Recent Approaches in Handwriting Recognition with Markovian Modelling and Recurrent Neural Networks. In: Recent Advances of Neural Network Models and Applications. Springer, 2014, pp. 261267.

[4] J Mantas. An overview of character recognition methodologies. In: Pattern recognition 19.6 (1986), pp. 425430.

[5] Talaat S El-Sheikh and Ramez M Guindi. Computer recognition of Arabic cursive scripts. In: Pattern Recognition 21.4 (1988), pp. 293302.

[6] Qi Tian et al. Survey: Omni font-printed character recognition. In: Visual Communications,91, Boston, MA. International Society for Optics and Photonics. 1991, pp. 260268.

[7] Shunji Mori, Ching Y Suen, and Kazuhiko Yamamoto. Historical review of OCR research and development. In: Proceedings of the IEEE 80.7 (1992), pp. 10291058.

[8] Amal Ramzi and Ammar Zahary. Online Arabic handwritten character recognition using online-offline feature extraction and backpropagation neural network. In: Advanced Technologies for Signal and Image Processing (ATSIP),2014 1st International Conference on. IEEE. 2014, pp. 350355

[9] Theodore Bluche, Hermann Ney, and Christopher Kermorvant. Feature extraction with convolutional neural networks for handwritten word recognition. In:Document Analysis and Recognition (ICDAR), 2013 12th International Confer- ence on. IEEE. 2013, pp. 285289.

[10] Amir Bahador Bayat. Recognition of Handwritten Digits Using Optimized Adaptive Neuro-Fuzzy Inference Systems and Effective Features. In: Journal of Pattern Recognition and Intelligent Systems.

[11] DK Patel, T Som, and Manoj Kumar Singh. Multiresolution technique to handwritten English character recognition using learning rule and Euclidean distance metric. In: Signal Processing and Communication (ICSC), 2013 International Conference on. IEEE. 2013, pp. 207212.

[12] Simone Marinai, Marco Gori, and Giovanni Soda. Artificial neural networks for document analysis and recognition. In: Pattern Analysis and Machine Intelligence, IEEE Transactions on 27.1 (2005), pp. 2335.

[13] Vassilis Papavassiliou et al. Handwritten document image segmentation into text lines and words. In: Pattern Recognition 43.1 (2010), pp. 369377

[14] SA Angadi and MM Kodabagi. A Robust Segmentation Technique for Line, Word and Character Extraction from Kannada Text in Low Resolution Display Board Images. In: Signal and Image Processing (ICSIP), 2014 Fifth Interna-tional Conference on. IEEE. 2014, pp. 4249.

[15] R Indra Gandhi and K Iyakutti. An attempt to recognize handwritten Tamil character using Kohonen SOM. In: Int. J. Advanced Network. Appl 1.3 (2009),pp. 188192

[16] J Venkatesh and C Sureshkumar. Tamil Handwritten Character Recognition Using Kohonons Self Organizing Map. In: the proceedings of IJCSNS International Journal of Computer Science and Network Security 9.12 (2009), pp. 156161.

[17] C Suresh Kumar and T Ravichandran. Handwritten Tamil character recognition using RCS algorithms. In: Int. J. of Computer Applications,(0975-8887) volume-8-no 8 (2010)

[18] Xuewen Wang, Xiaoqing Ding, and Changsong Liu. Optimized Gabor filter based feature extraction for character recognition. In: Pattern Recognition, 2002. Proceedings. 16th International Conference on. Vol. 4. IEEE. 2002, pp. 223226.

[19] Kai Ding et al. A comparative study of Gabor feature and gradient feature for handwritten Chinese character recognition. In: Wavelet Analysis and Pattern Recognition, 2007. ICWAPR07. International Conference on. Vol. 3. IEEE.2007, pp. 11821186.

[20] Cheng-Lin Liu, Masashi Koga, and Hiromichi Fujisawa. Gabor feature extraction for character recognition: comparison with gradient feature. In: Document Analysis and Recognition, 2005. Proceedings. Eighth International Conference on. IEEE. 2005, pp. 121125.

[21] Zahra Imani et al. Offline handwritten Farsi cursive text recognition using hidden Markov models. In: Machine Vision and Image Processing (MVIP), 2013 8th Iranian Conference on. IEEE. 2013, pp. 7579.

[22] Richa Goswami and OP Sharma. A Review on Character Recognition Techniques. In: International Journal of Computer Applications 83.7 (2013), pp. 1823.

[23] Jayshree Rajesh Prasad and Uday V Kulkarni. Gujrati Character Recognition Using Adaptive Neuro Fuzzy Classifier. In: Electronic Systems, Signal Processing and Computing Technologies (ICESC), 2014 International Conference on. IEEE. 2014 pp. 402407.

[24] Bingyu Chi and Youbin Chen. Chinese Handwritten Legal Amount Recognition with HMM-Based Approach. In: Document Analysis and Recognition (ICDAR), 2013 12th International Conference on. IEEE. 2013, pp. 778782.

[25] Arifur Rahaman et al. Analysis on handwritten Bangla character recognition using ANN. In: Informatics, Electronics \& Vision (ICIEV), 2014 International Conference on. IEEE. 2014, pp. 15.

[26] Dewi Nasien, Habibollah Haron, and Siti Sophiayati Yuhaniz. Support Vector Machine (SVM) for English Handwritten Character Recognition. In: Computer Engineering and Applications (ICCEA), 2010 Second International Conference on. Vol. 1. IEEE. 2010, pp. 249252.

[27] Nisha Sharma, Bhupendra Kumar, and Vandita Singh. Recognition of offline hand printed English Characters, Numerals and Special Symbols. In: Confluence The Next Generation Information Technology Summit (Confluence), 2014 5th International Conference-. IEEE. 2014, pp. 640645.

[28] Jawad H AlKhateeb and Marwan Alseid. DBN-Based learning for Arabic handwritten digit recognition using DCT features. In: Computer Science and Information Technology (CSIT), 2014 6th International Conference on. IEEE. 2014, pp. $222-226$. 